\title{
Multimodal imaging of right coronary artery to left ventricle fistula complicated by large coronary aneurysm
}

\author{
Takao Konishi ${ }^{1,2}$, Tadashi Yamamoto ${ }^{1}$, Masato Hayakawa ${ }^{3}$, \\ Shizuko Iwasa ${ }^{3}$, Hiroyuki Tsukui ${ }^{3}$, Shinya Tanaka ${ }^{2}$ \\ ${ }^{1}$ Department of Cardiology, Hokkaido Cardiovascular Hospital, Sapporo, Japan \\ ${ }^{2}$ Department of Cancer Pathology, Hokkaido University, Graduate School of Medicine, Sapporo, Japan \\ ${ }^{3}$ Department of Cardiovascular Surgery, Hokkaido Cardiovascular Hospital, Sapporo, Japan
}

An asymptomatic 45-year-old woman presented with incidentally detected diastolic murmur. The initial echocardiogram showed an abnormal blood flow in the left ventricle (LV) (Fig. 1A). Cardiac computed tomography revealed a right coronary artery
(RCA) to LV fistula complicated by a coronary artery aneurysm (CAA) (Fig. 1B, C; Suppl. Video S1). Coronary angiograms (Fig. 1D; Suppl. Video S2) followed by a coronary flow measurement (Fig. 1E) confirmed a brisk blood flow of $91 \mathrm{~cm} / \mathrm{s}$ through

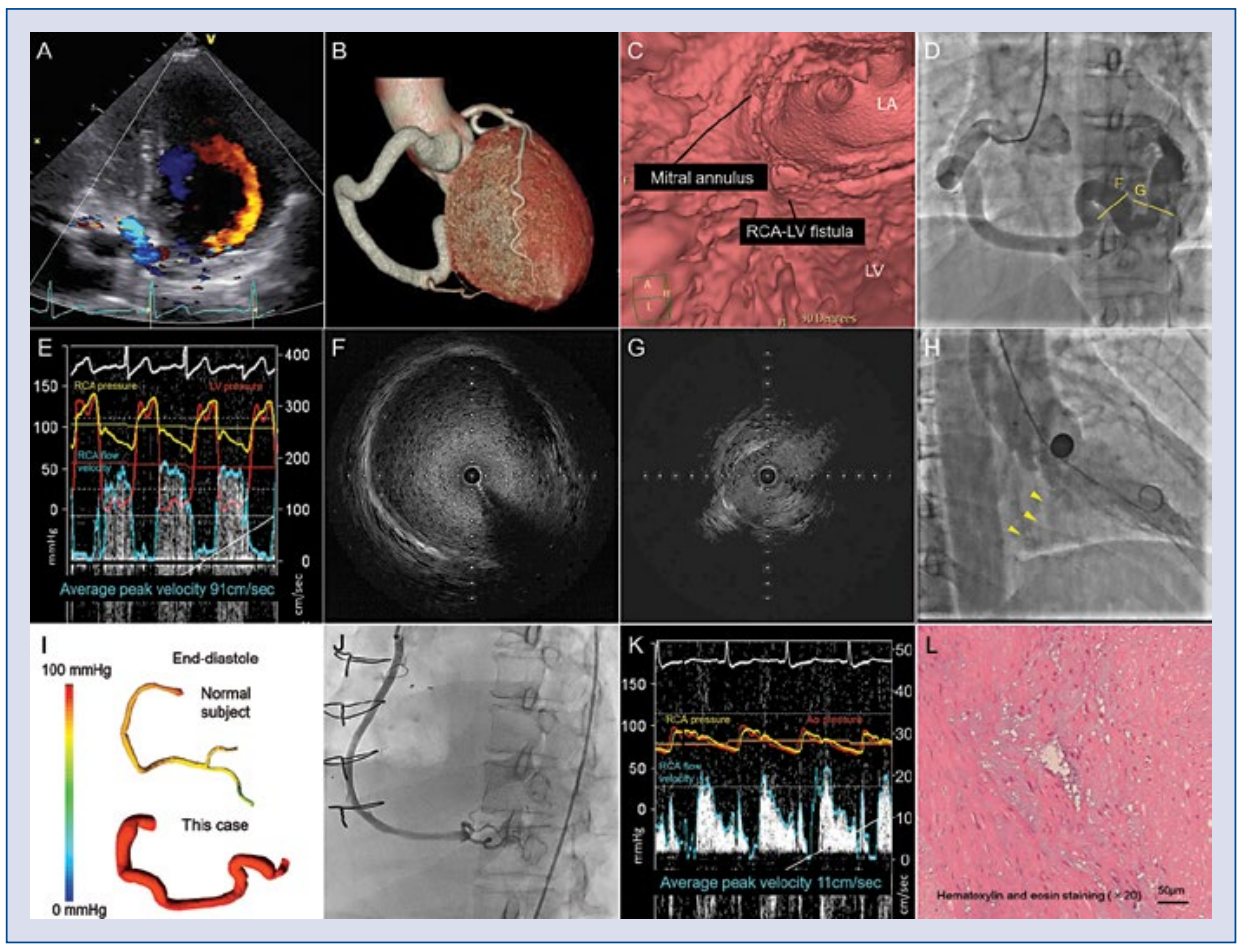

Figure 1. A. The initial echocardiogram; B, C. Cardiac computed tomography; D. Coronary angiograms; E. Coronary flow measurement; F, G. Intravascular ultrasound study; H. Ventriculogram; I. Computational fluid dynamics; J. Bypass graft angiography; K. Coronary flow measurement after surgery; L. Histopathology.

Address for correspondence: Takao Konishi, MD, Department of Cardiology, Hokkaido Cardiovascular Hospital, 1-30, West 13, South 27, Chuou-ku, Sapporo, Japan, 064-8622, e-mail: takaokonishi0915@gmail.com

Received: 11.12.2017 Accepted: 29.12.2017 
CAA. An intravascular ultrasound study showed that the widest diameter of CAA was $13 \mathrm{~mm}$ (Fig. 1F) and the exit of CAA to LV shrank but remained open during systole (Fig. 1G). A left ventriculogram showed opacified blood flowing from LV into CAA during systole after occlusion test by a $10-\mathrm{mm}$ balloon (Fig. 1H; Suppl. Video S3). The electrocardiogram and echocardiogram showed no ischemic changes despite 20-min occlusion, suggesting that the RCA territory was fed by regurgitant flow from LV. The analysis of computational fluid dynamics, using ANSYS 15.0 software (ANSYS Inc., USA), showed that the CAA was exposed to high blood pressure during entire cardiac cycle (Fig. 1I; Suppl. Video S4). To prevent the late complications including rupture, surgical ligation of the CAA and coronary artery bypass grafting were performed instead of transcatheter embolization (Fig. 1J). The blood flow of RCA was normalized to $11 \mathrm{~cm} / \mathrm{s}$ (Fig. 1K). Histopathologically, the media of CAA showed prominent myxoid degeneration, which suggested the presence of long-term pressure loading (Fig. 1L).

Conflict of interest: None declared 